\title{
VERY LARGE ARRAY LIMITS FOR INTERMEDIATE-MASS BLACK HOLES IN THREE GLOBULAR CLUSTERS
}

\author{
F. N. Bash ${ }^{1}$, K. Gebhardt ${ }^{1}$, W. M. Goss ${ }^{2}$, and P. A. Vanden Bout ${ }^{3}$ \\ ${ }^{1}$ Department of Astronomy, University of Texas at Austin 1 University Station C1400, Austin, TX 78712, USA; fnb@astro.as.utexas.edu and \\ gebhardt@astro.as.utexas.edu \\ 2 National Radio Astronomy Observatory, P.O. Box 0, Socorro NM 878701, USA; mgoss@nrao.edu \\ ${ }^{3}$ National Radio Astronomy Observatory, 520 Edgemont Road, Charlottesville, VA 22903, USA; pvandenb@nrao.edu \\ Received 2007 July 11; accepted 2007 September 29; published 2007 December 7
}

\begin{abstract}
The observational evidence for central black holes in globular clusters has been argued extensively, and their existence has important consequences for both the formation and evolution of the cluster. Most of the evidence comes from dynamical arguments, but the interpretation is difficult, given the short relaxation times and old ages of the clusters. One of the most robust signatures for the existence of a BH is radio and/or X-ray emission. We observed three globular clusters: NGC6093 (M80), NGC6266 (M62), and NGC7078 (M15), with the Very Large Array (VLA) in the A and C configuration with a 3- $\sigma$ noise of 36, 36, and $25 \mu \mathrm{Jy}$, respectively. We find no statistically significant evidence for radio emission from the central region for any of the three clusters. NGC6266 shows a $2-\sigma$ detection. It is difficult to infer a mass from these upper limits due to uncertainty about the central gas density, accretion rate, and accretion model.
\end{abstract}

Key words: accretion, accretion disks - black hole physics - globular clusters: general - radio continuum: general

Online-only material: color figures

\section{INTRODUCTION}

Although we do not understand how the nuclei of galaxies form or why they have black holes (BHs) at their centers, the correlation between the $\mathrm{BH}$ mass and bulge velocity dispersion does shed light on their formation and evolutionary histories (Gebhardt et al. 2000; Ferrarese and Merritt 2000). A number of different theories (e.g., Silk \& Rees 1998; Haehnelt \& Kauffmann 2000; Robertson et al. 2006) predict a BH massbulge-velocity-dispersion relation, although they predict different slopes and intercepts for this relation. Exploration of the extreme ends of this relationship will help illuminate the underlying physical model, and in this paper we focus on the low-mass end. BHs at the low end of the relations, with masses between 100 and $10^{6} M_{\odot}$, are generally referred to as intermediate-mass black holes (IMBHs). There is a significant evidence that $\mathrm{BH}$ masses from $10^{5}$ to $10^{6} M_{\odot}$ exist from the work of Barth et al. (2005) and Greene \& Ho (2006). To go to yet smaller BH masses, an extrapolation of the correlation between the $\mathrm{BH}$ mass and stellar velocity dispersion suggests studying stellar systems with velocity dispersions of $10-20 \mathrm{~km} \mathrm{~s}^{-1}$. These dispersions are characteristic of globular clusters. Whether the existence of BHs in globular clusters could shed light on the formation and correlations of supermassive BHs is unknown, but clearly there is a possibility. Furthermore, the existence of massive $\mathrm{BHs}$ in clusters will have a significant effect on the cluster evolution. Thus, quantifying the demographics of BHs in clusters may be related to how the supermassive BHs grow, and will definitely yield useful information about the evolution of clusters.

Theoretical work suggests that we might expect IMBHs at the centers of steller systems (Ebisuzaki et al. 2001; Portegies Zwart \& McMillian 2002; Miller \& Hamilton 2002), although it appears to be difficult to make BHs more massive than $100 M_{\odot}$. Gurkan et al. (2004) suggested that IMBHs may be easy to form through runaway collisions with massive stars.
Discoveries of BHs in globular clusters have been claimed - G1 in M31 (Gebhardt et al. 2002) and M15 (van der Marel et al. 2002). In fact, the M15 claim has been made for the past 30 years, starting with the result of Newell et al. (1976) and subsequently challenged by Illingworth \& King (1977). The basic issue is being able to distinguish a rise in the central mass-to-light ratio being due to either a $\mathrm{BH}$ or the expected stellar remnants (neutron stars, massive white dwarfs and solar mass BHs). The most recent M15 result has been challenged by Baumgardt et al. (2003a). The result in G1 has also been challenged by Baumgardt et al. (2003b) but Gebhardt et al. (2005) included additional data and analysis that support the $\mathrm{BH}$ interpretation.

There have been two further observations which strongly support the existence of a BH in G1. Trudolyubov \& Priedhorsky (2004) measured X-rays from G1 using the Chandra Observatory, centered at within $2^{\prime \prime}$ of the center of G1. Subsequently, Pooley \& Rappaport (2006) suggested that the Xray emission is from accretion onto a $\mathrm{BH}$, and Maccarone \& Koerding (2006) pointed out that if a BH is present then a $30 \mu \mathrm{Jy}$ radio source may be expected. The most significant observation comes from Ulvestad et al. (2007) who found a $28 \mu \mathrm{Jy}(4.5-\sigma)$ emission centered at G1. Other interpretations are a pulsar wind or a planetary nebula. The pulsar wind seems unlikely given the age of G1 and the point-like radio source (an old pulsar would have a large size). A planetary nebula would show optical emission lines which are not seen in the Hubble Space Telescope (HST) or Keck spectra of Gebhardt et al. (2005).

Other studies of the existence of BHs in globular clusters have been less compelling. Colpi et al. (2003) used indirect dynamical arguments to suggest a few hundred solar mass BHs in NGC 6752. McLaughlin et al. (2006) provided an estimate of $\mathrm{BH}$ in 47 Tuc of $900 \pm 900 M_{\odot}$ To date, there are no published upper limits of $\mathrm{BH}$ masses that are significantly below that expected from an extrapolation of the correlation between the $\mathrm{BH}$ mass and stellar velocity dispersion. 
Table 1

8.6 GHz Flux Density Values

\begin{tabular}{|c|c|c|c|c|}
\hline & (2) & (3) & (4) & (5) \\
\hline (1) & $M_{\mathrm{BH}}$ & $M_{\mathrm{BH}}$ & Distance & Flux density \\
\hline Cluster & Maccarone $\left(M_{\odot}\right)$ & This paper $\left(M_{\odot}\right)$ & $\mathrm{kpc}$ & $\mu \mathrm{Jy}$ \\
\hline NGC6093 (M80) & $\ldots$ & 1600 & 8 & $2 \times 10^{3}-10^{5}$ \\
\hline NGC6266 (M62) & 450 & 3000 & 6 & $3 \times 10^{3}-10^{5}$ \\
\hline NGC7078 (M15) & 440 & 1000 & 10 & $1 \times 10^{3}-10^{5}$ \\
\hline
\end{tabular}

While the dynamical arguments strongly support the $\mathrm{BH}$ interpretation in at least $\mathrm{G} 1$, the radio emission provides a clear and obvious result. Unfortunately, it is difficult to predict the radio emission from a given $\mathrm{BH}$ mass. The next step is to explore other globular clusters with a similar setup and deep exposures.

\section{TARGET SAMPLE AND RADIO FLUX DENSITY PREDICTIONS}

We selected three globular clusters for observation. First, using the stellar velocity dispersion at the center of M15 (NGC7078) suggests a revision of the mass of the $\mathrm{BH}$ at the cluster's center to $1000 M_{\odot}$, higher than Maccarone's assumed value of $440 M_{\odot}$, making M15 a promising candidate. Second, noting that Baumgardt et al. (2005) argue that highly centrally condensed globular clusters, as seen from their luminosity profiles, are unlikely to harbor central IMBHs, we selected two globular clusters with large cores that are more likely to have central BHs. These clusters, NGC6093 and NGC6266, also have large central stellar velocity dispersions.

In order to predict the radio flux density, the first step is to use an expected $\mathrm{BH}$ mass. The $\mathrm{BH}$ masses can be estimated using an extrapolation of the correlations seen in galaxies, namely either the $\mathrm{BH}$ mass/velocity dispersion or the $\mathrm{BH}$ mass/galaxy bulge luminosity relations.

A precise prediction of the expected radio flux densities based on the BH mass is quite uncertain. Merloni et al. (2003) use radio flux densities, X-ray luminosities, and measured $\mathrm{BH}$ masses from both Galactic and extragalactic BHs to derive a fundamental plane for the three parameters. They argue that using any one parameter to predict another is quite uncertain. Unfortunately, X-ray luminosities do not exist for the three clusters studied here. Furthermore, the study of Merloni et al. does not include any BHs with masses from 10 to $10^{6}$, making any use of the fundamental plane suspect for the three globular clusters. Therefore, instead of directly using the expected $\mathrm{BH}$ mass and the measured X-ray luminosity to predict the radio flux density, we simply use the location between the $10^{6} M_{\odot} \mathrm{BHs}$ and the galactic BHs in the fundamental plane. In this region, the expected $5 \mathrm{GHz}$ radio power ranges from $10^{30}$ to $10^{32} \mathrm{ergs} \mathrm{s}^{-1}$. Indeed, for the G1 radio emission from Ulvestad et al. (2007) corresponds to $10^{32} \mathrm{ergs} \mathrm{s}^{-1}$, which is consistent with the measured $\mathrm{BH}$ mass of $2 \times 10^{4}$ from Gebhardt et al. (2005). Thus, in order to predict the expected flux densities, we adopt this range in radio power and use the known distances of the globular clusters. A significant assumption in these estimates is that the physical conditions are similar; if, for example, the gas density were much lower in globular clusters, the predicted radio power would be much less.

Alternatively, Maccarone (2004) estimated the expected radio emission based on the expected gas density and the correlation of Merloni et al. (2003). The gas density in the cluster come from the estimate of Freire et al. (2001) who used differences in column densities measured from pulsars in the front and back sides in the globular cluster 47 Tuc. While there is no reason to expect similar gas densities from cluster to cluster, it is the best measure we have of gas density in a cluster and therefore we adopt that value. Maccarone (2004) further assumed that the $\mathrm{BH}$ is accreting the intra-cluster gas at 0.1 and $1 \%$ of the Bondi accretion rate. He assumes the $\mathrm{BH}$ mass to be $0.1 \%$ of the globular cluster mass, which he estimates from the cluster's total luminosity and an assumed mass-to-light ratio, and computes the expected $5 \mathrm{GHz}$ flux density from the vicinity of the central $\mathrm{BH}$ for 15 globular clusters. Six of the globular clusters in Maccarone's list lie north of the southern declination limit of the Very Large Array ${ }^{4}$ (VLA) and have an estimated $5 \mathrm{GHz}$ flux density of $40 \mu \mathrm{Jy}$ or greater (at $1 \%$ of the Bondi rate). We searched the VLA archive for observations of the centers of these clusters with noise levels low enough to have allowed a detection at Maccarone's predicted levels. No VLA archive data were found which had the required sensitivity.

There have been two similar studies for the one presented here. Maccarone et al. (2005) provided upper limits for omega Cen using ATCA observations and for M15 from archival VLA observations. De Rijcke et al. (2006) provided upper limits for 47 Tuc and NGC 6397 based on ATCA observations.

Table 1 shows the computed flux densities for NGC6266, NGC7078, and NGC6093 at a frequency of $8.6 \mathrm{GHz}$. We assume a spectral index, $\alpha=-0.7$, to provide the predicted fluxes at $8.6 \mathrm{GHz}$, the frequency of our observations. Our BH masses come from the $\mathrm{BH} /$ sigma correlation (shown in the second column), and we also report those from Maccarone based on luminosity (the first column).

\section{OBSERVATIONS}

Source positions, integration times on source, beam dimensions and position angles, and our $3-\sigma$ limits are given in Table 2.

We used the position of the center of M15 determined by Noyola \& Gebhardt (2006) using the optical surface brightness profile from the Hubble Space Telescope (HST), which are good to less than $1^{\prime \prime}$. We observed that position using the VLA for 7.5 hours on 2004 October 13 in the A configuration at $8.6 \mathrm{GHz}(\lambda 3.5 \mathrm{~cm})$, where the VLA has its maximum sensitivity. The resulting map has an rms noise level of $8.5 \mu \mathrm{Jy} / \mathrm{beam}$ and covers $\approx 1$ arcmin positioned at the center of M15. We clearly see the source AC211 reported by Johnston et al. (1991) about 1.5 arcsec northwest of the cluster center at with a peak flux density on our map of $144 \mu \mathrm{Jy}$. We do not detect the other known low mass X-ray binary, M15 X-2 (White \& Angelini $2001)$, even at $1-\sigma$. The image also contains the planetary nebula K 648, for which we get R.A. $21^{\mathrm{h}} 29^{\mathrm{m}} 59^{\mathrm{s}} .39$, decl. $12^{\circ} 10^{\prime} 26^{\prime \prime} 46$ (J2000). The measured flux density is $4.2 \pm 0.2 \mathrm{mJy}$ and the deconvolved size is $1.5 \times 0.7$ arcsec. We do not see the pulsar

\footnotetext{
4 The VLA is operated by the National Radio Astronomy Observatory, which is a facility of the National Science Foundation, operated under cooperative agreement by Associated Universities, Inc.
} 


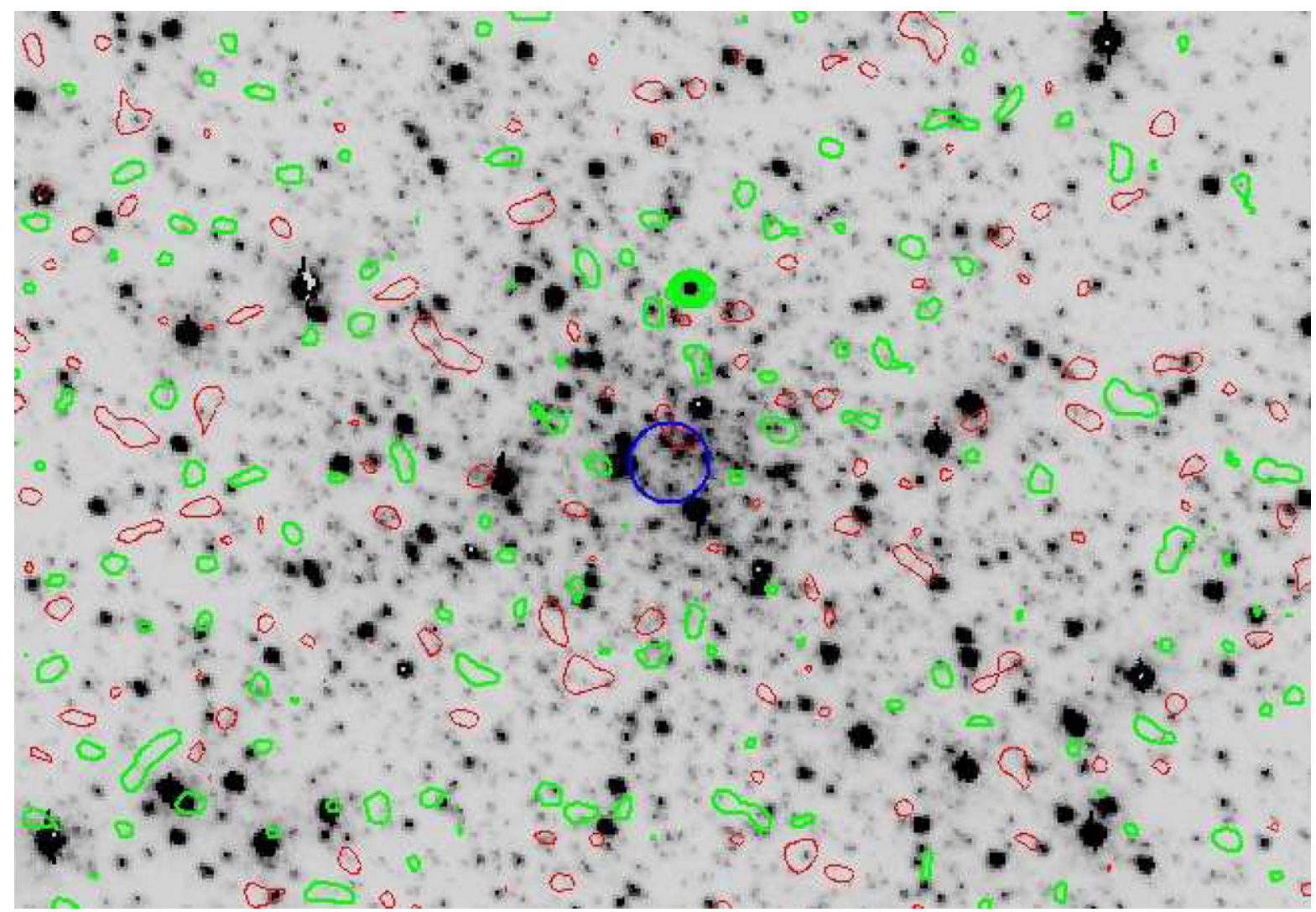

Figure 1. HST optical image of M15 overlayed with the VLA contours of the central 10". Positive 1- $\sigma, 2-\sigma$, and 3- $\sigma$ noise contours $(8.5,17$, and $25.5 \mu \mathrm{Jy})$ are shown in green and negative contours are shown in red. The blue circle marks the center determined from Noyola \& Gebhardt (2006), with a diameter of 0.5". North is up and east to the left. The radio source, AC211, is easily seen just north of the center. The other known X-ray source, M15 X-2, is not detected.

(A color version of this figure is available in the online journal)

Table 2

Observations

\begin{tabular}{|c|c|c|c|c|c|}
\hline $\begin{array}{l}\text { (1) } \\
\text { Cluster }\end{array}$ & $\begin{array}{c}(2) \\
\text { RA } \\
\text { J2000 }\end{array}$ & $\begin{array}{c}\text { (3) } \\
\text { Decl. } \\
\text { J2000 }\end{array}$ & $\begin{array}{c}\text { (4) } \\
\text { Integration } \\
\text { (hours) }\end{array}$ & $\begin{array}{c}\text { (5) } \\
\text { Beam; position angle } \\
\text { (arcseconds; deg) }\end{array}$ & $\begin{array}{c}(6) \\
3-\sigma \text { limit } \\
(\mu \mathrm{Jy})\end{array}$ \\
\hline NGC6093 & $16: 17: 05.00$ & $-22: 59: 47.3$ & 7.0 & $3.9 \times 2.3 ;-6$ & 36 \\
\hline NGC6266 & 17:01:12.96 & $-30: 06: 46.2$ & 7.0 & $4.7 \times 2.2 ;-6$ & 36 \\
\hline NGC7078 & 21:29:58.35 & $+12: 10: 01.5$ & 6.5 & $0.2 \times 0.2 ;-76$ & 25 \\
\hline
\end{tabular}

PSR 2127+12A or any other point source near the center of M15 at or above a level of $25 \mu \mathrm{Jy} / \mathrm{beam}$.

The positions of the centers of NGC6093 and NGC6266 also come from Noyola \& Gebhardt (2006) (with a similar accuracy of around $\left.1^{\prime \prime}\right)$. These clusters were observed on 2005 August 11,20 , and 26 using the VLA in the C configuration at $8.6 \mathrm{GHz}$. Each map has an rms noise of $12 \mu \mathrm{Jy} / \mathrm{beam}$. These maps cover an area of 5.4 arcmin centered at each cluster's center. No radio source is seen at or above a level of $36 \mu \mathrm{Jy} / \mathrm{beam}$ near either cluster's center. In the case of NGC6093 a source is detected 82 arcsec to the SE of the cluster center at R.A. $16^{\mathrm{h}} 17^{\mathrm{m}} 05^{\mathrm{s}} .00$, decl. $-22^{\circ} 59^{\prime} 47^{\prime \prime} 3$ (J2000) with a flux density of $0.32 \pm$ $0.05 \mathrm{mJy}$. The distance from the cluster center makes it unlikely that this source is related to NGC6266. No source is seen convincingly in the image of NGC6266.

Figures 1-3 plot the HST optical images (as used in Noyola $\&$ Gebhardt) with the VLA radio contours. None of the three clusters shows a significant detection at $8 \mathrm{GHz}$ at the cluster center. However, NGC 6266 (M62) shows about a 2- $\sigma$ peak at the center.

\section{DISCUSSION}

Failure to detect radio radiation at $8.6 \mathrm{GHz}$ from the centers of the three globular clusters does not prove that no globular clusters have IMBHs at their centers. Besides not having a $\mathrm{BH}$, other interpretations include (1) accretion by the $\mathrm{BH}$ could be episodic and we happened to observe the BHs in an "offstate," (2) the gas density could be much lower compared to galaxies, (3) the radiative efficiency may be lower than assumed (although the assumed efficiencies are already quite low), (4) or the accretion model may not be adequate in general. We would predict, using the relation of Merloni et al. (2003) or using standard accretion models and gas density estimates (as done in Maccarone 2004), that we should have detected radio radiation at $8.6 \mathrm{GHz}$ if the accretion is steady and the accretion rate times the Bondi rate is $10^{-4} \times$ or higher. We would not have been able to detect the flux density predicted by a rate of $10^{-5} \times$ or less. Ulvestad et al. (2007) estimated the fraction of the Bondi rate of just below $1 \%$ for $\mathrm{G} 1$, but it is difficult to interpret due to the unknown radiative efficiency. For 


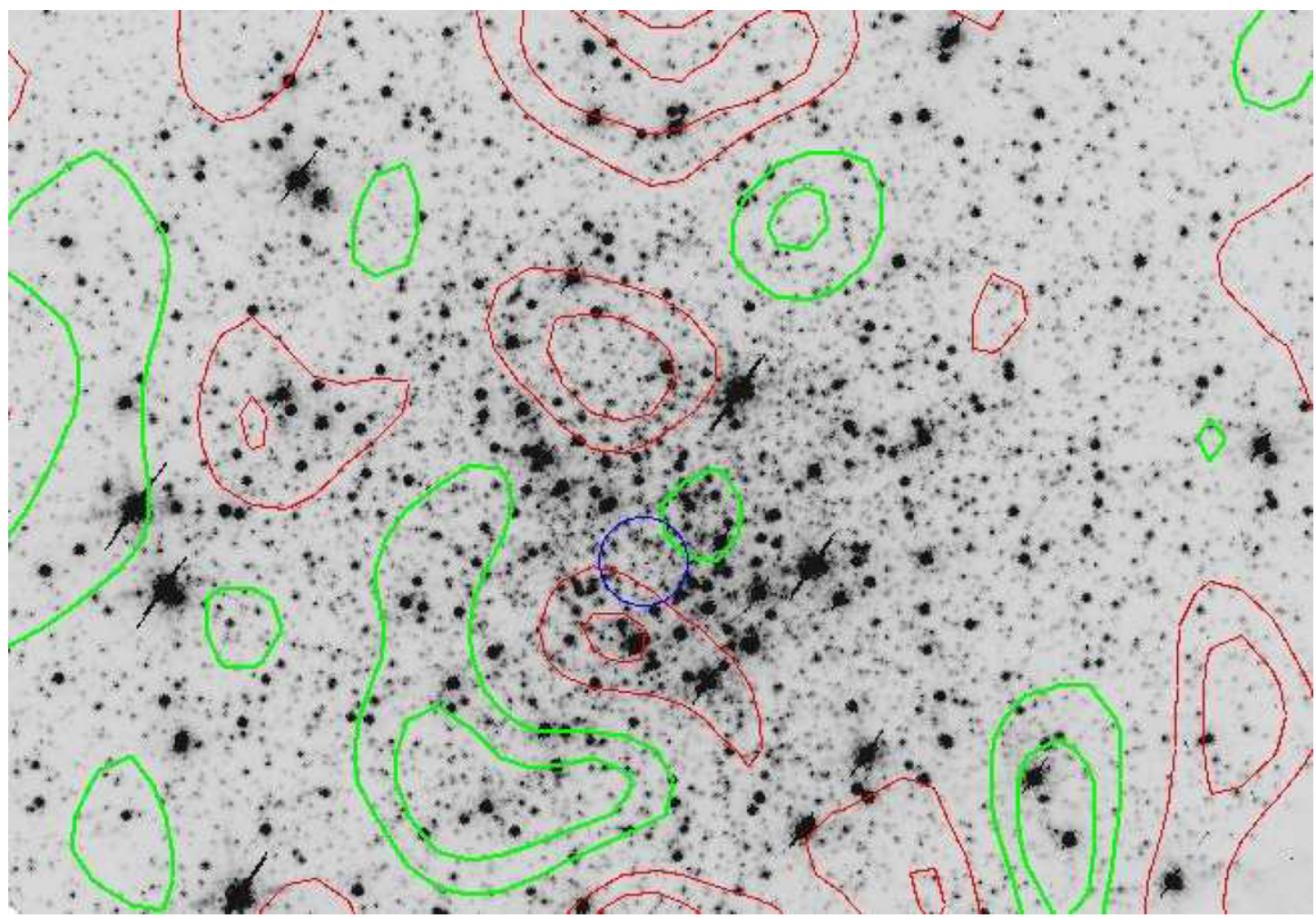

Figure 2. HST optical image of NGC 6093 (M80) overlayed with the VLA contours of the central 10". Positive 1- $\sigma, 2-\sigma$, and 3- $\sigma$ noise contours (12, 24, and 36 $\mu \mathrm{Jy})$ are shown in green and negative contours are shown in red. The blue circle marks the center determined from Noyola \& Gebhardt (2006), with a diameter of $1^{\prime \prime}$. North is up and east to the left.

(A color version of this figure is available in the online journal)

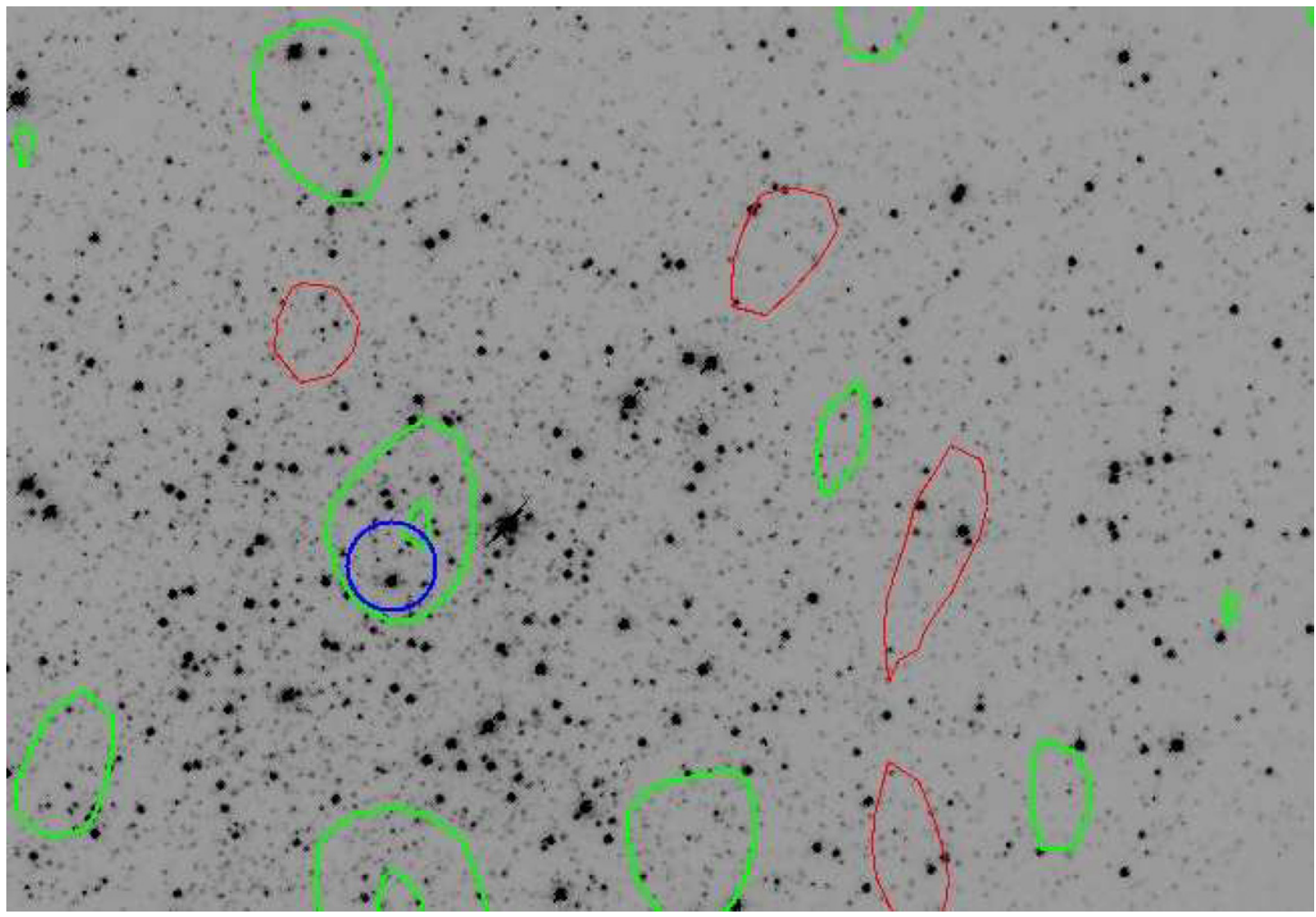

Figure 3. HST optical image of NGC 6266 (M62) overlayed with the VLA contours of the central 10". Positive 1- $\sigma$, 2- $\sigma$, and 3- $\sigma$ noise contours (12, 24, and $36 \mu \mathrm{Jy})$ are shown in green and negative contours are shown in red. The blue circle marks the center determined from Noyola \& Gebhardt (2006), with a diameter of $1^{\prime \prime}$. North is up and east to the left. There is about a $2-\sigma$ positive $8 \mathrm{GHz}$ signal at the center.

(A color version of this figure is available in the online journal) 
galactic $\mathrm{BHs}$, the radiative efficiencies appear to vary greatly with some lower than $10^{-5}$ (Lowenstein et al. 2001), although they are consistent with rates of around $10 \%$ of the Bondi rate.

Models which predict $8.6 \mathrm{GHz}$ flux densities from the central $\mathrm{BHs}$ in globular clusters above about $25 \mu \mathrm{Jy} /$ beam can be tested with the VLA currently. The EVLA should produce, for continuum observations, a sensitivity improvement of about a factor of 15 , making $8.6 \mathrm{GHz}$ flux densities above about $2 \mu \mathrm{Jy} / \mathrm{beam}$ detectable.

\section{REFERENCES}

Barth, A., Greene, J., \& Ho, L. C. 2005, ApJ, 619, L151

Baumgardt, H., Hut, P., Makino, J., McMillan, S., \& Portegies Zwart, S. 2003a, ApJ, 582, L21

Baumgardt, H., Makino, J., \& Hut, P. 2005, ApJ, 620, 238

Baumgardt, H., Makino, J., Hut, P., \& Portegies Zwart, S. 2003b, ApJ, 589, L25

Colpi, M., Mapelli, M., \& Possenti, A. 2003, ApJ, 599, 1260

De Rijcke, S., Buyle, P., \& Dejonghe, H. 2006, MNRAS, 368, 43

Ebisuzaki, T., et al. 2001, ApJ, 562, L19

Ferrarese, L., \& Merritt, D. 2000, ApJ, 539, L9

Freire, P., Kramer, M., Lyne, A., Camilo, F., Manchester, R., \& D’Amico, N. 2001, ApJ, 557, L105

Gebhardt, K., et al. 2000, ApJ, 539, L13
Gebhardt, K., Rich, R. M. R., \& Ho, L. C. 2002, ApJ, 578, L41 Gebhardt, K., Rich, R. M. R., \& Ho, L. C. 2005, ApJ, 634, 1093 Greene, J., \& Ho, L. C. 2006, ApJ, 641, L21

Gurkan, M., Freitag, M., \& Rasio, F. 2004, ApJ, 604, 632

Haehnelt, M. G., \& Kauffmann, G. 2000, MNRAS, 318, L35

Illingworth, G., \& King, I. 1977, ApJ, 218, L109

Jonhston, H., Kulkarni, S., \& Goss, W. M. 1991, ApJ, 382, L89

Lowenstein, M., Mushotsky, R., Angelini, L., Arnaud, K., \& Quataert, E. 2001, ApJ, 555, L21

Maccarone, T. 2004, MNRAS, 351, 1049

Maccarone, T., \& Koerding, 2006, Astron. Geophys., 47, 29

Maccarone, T., Fender, R., \& Tzioumis, A. 2005, Ap\&SS, 300, 247

McLaughlin, D., Anderson, J., Meylan, G., Gebhardt, K., Pryor, C., Minniti, D., \& Phinney, S. 2006, ApJS, 166, 249

Merloni, A., Heinz, S., \& di Matteo, T. 2003, MNRAS, 345, 1057

Miller, M. C., \& Hamilton, D. P. 2002, MNRAS, 330, 232

Newell, B., Da Costa, G., \& Norris, J. 1976, ApJ, 208, L55

Noyola, E., \& Gebhardt, K. 2006, AJ, 132, 447

Pooley, D., \& Rappaport, S. 2006, ApJ, 644, L45

Portegies Zwart, S., \& McMillan, S. 2002, ApJ, 576, 899

Robertson, B., Hernquist, L., Cox, T., Di Matteo, T., Hopkins, P., Martini, P., \& Springel, V. 2006, ApJ, 641, 90

Silk, J., \& Rees, M. J. 1998, A\&A, 331, L1

Trudolyubov, S., \& Priedhorsky, W. 2004, ApJ, 616, 821

Ulvestad, J., Greene, J., \& Ho, L. C. 2007, ApJ, 661, 151

van der Marel, R. P., Gerssen, J., Guhathakurta, R., Peterson, R., \& Gebhardt, K. 2002, AJ, 124, 3255

White, N., \& Angelini, L. 2001, ApJ, 561, L101 